\title{
Avaliação nutricional de Butiá (Butia yatai) processado
}

\author{
Josué Schneider Martins ${ }^{1 *}$, Eduardo Madruga Melo ${ }^{2}$, Lucas Prestes Fallavena ${ }^{1}$ e Plinho \\ Franscisco Hertz ${ }^{1}$
}

No Rio Grande do Sul, destaca-se a relevância socioambiental de diferentes espécies de butiá (butiá yatai). Este trabalho teve como objetivo avaliar como o processo de despolpa de butiá interfere, ao longo de três meses de armazenamento, na composição do alimento, particularmente no valor calórico, no teor de fibras e na estabilidade do ácido ascórbico. Os frutos de butiá foram coletados e analisados entre os meses de março e junho de 2017, analisando-se parâmetros físico-químicos e centesimais da fruta e da polpa de butiá. Do ponto de vista físico-químico, a polpa, quando comparada à fruta, apresenta maior teor de umidade, de açúcares dissolvidos e teor de ácido cítrico. A polpa apresentou aumento de $24,5 \%$ na quantidade energética total, porém, quando se trata das fibras alimentares totais, houve redução de 47,9\%. O teor de ácido ascórbico diminuiu 44,6\% durante o período estudado. Tanto a fruta como a polpa apresentaram alto teor de fibras alimentares. Portanto, foi possível identificar que o processo de beneficiamento do butiá acarreta em mudanças nas características da fruta para a polpa, mas ambos os alimentos podem ser considerados nutritivos para compor a base da alimentação regional.

Palavras-chave: Processamento. Fruta Nativa. Guia Alimentar.

\section{Nutritional evaluation of Butia (Butia yatai) processed}

In the state of Rio Grande do Sul, Brazil, it is important to highlight the socioenvironmental relevance of different butia species. The objective of this work was to evaluate how butia (Butia yatai) pulping process interferes, within three months of storage, in the food composition - particularly caloric value, fiber content and the stability of ascorbic acid. Butia fruits were collected and analyzed between March and June 2017, analyzing physico-chemical properties and centesimal composition of butia fruit and pulp. Regarding the physico-chemical characteristics, the pulp, when compared to the fruit, presents a higher content of moisture, dissolved sugars and citric acid. Also, the pulp showed a $24.5 \%$ increase in the total energy content. However, when it comes to the total dietary fiber, there was a 47.9 $\%$ reduction. The ascorbic acid content decreased $44.6 \%$ during the studied period. Both fruit and pulp presented a high fiber content. Therefore, it was possible to identify that the fruit processing involves changes on the pulp characteristics, but both, the in nature fruit and processed, can be considered nutritious and of good assistance in the regional diet.

Keywords: Processing. Native Fruit. Food Guide for Brazilian Population.

1 Programa de Pós-Graduação em Ciência e Tecnologia de Alimentos (PPGCTA) da Universidade Federal do Rio Grande do Sul (UFRGS). Endereço para Correspondência: Av. Bento Gonçalves, 9500 - Campus do Vale - Prédio 43.212 - CEP: 91501970 - Porto Alegre - RS. Telefone: (54) 98444-2340

2 Graduando em Engenharia de Alimentos, Universidade Federal do Rio Grande do Sul 


\section{INTRODUÇÃO}

O consumo de frutas nativas pode ser fundamental para a saúde da sociedade como um todo. Se por um lado são alimentos que contém diversos nutrientes como fibras, vitaminas $\mathrm{e}$ minerais ${ }^{[1,2]}$, por outro, são produtos que podem integrar o conceito de "Dieta Sustentável" proposta pela $\mathrm{ONU}^{[3]}$ quando compõe sistemas produtivos que valorizem a biodiversidade e contribuam para a distribuição de renda. Além disso, a própria discussão a cerca do conceito de "alimentação saudável" tem sido discutida de modo amplo, unindo as questões nutricionais às ambientais e sociais ${ }^{[4]}$.

No Rio Grande do Sul, cabe destacar a relevância social e ambiental das diferentes espécies de butiá ${ }^{[1]}$. As frutas desta palmeira são importantes do ponto de vista histórico e cultural, pois existem relatos que diferentes povos indígenas habitualmente consumiam a fruta e a amêndoa, bem como econômico no sentido de que serve de base para diferentes produtos de artesanato e alimentícios ${ }^{[5,6]}$. $\mathrm{O}$ fruto do butiazeiro é utilizado de diferentes formas tradicionais como para a elaboração de sucos e doces, mas principalmente seu uso é reconhecido para a elaboração de licores ${ }^{[6]}$. Do ponto de vista econômico e ambiental também assumem grande importância, pois já despontam, junto ao açaí juçara (Euterpes edulis), como carros chefes da produção de frutas nativas do estado do $\mathrm{RS}^{[7]}$.

Com o objetivo de fomentar escolhas alimentares mais saudáveis, o Ministério da Saúde lançou em 2014 a segunda edição do Guia Alimentar para População Brasileira ${ }^{[8]}$ Este documento, como resultado de um debate acadêmico aprofundado ${ }^{[9,10]}$, apresenta diferentes princípios e diretrizes fundamentais para escolhas alimentares adequadas e saudáveis. Entre diferentes discussões levantadas neste documento está a recomendação de sempre dar preferência a alimentos in natura ou minimamente processados para compor a base alimentar da população em detrimento dos ultraprocessados.
Entretanto, o mesmo Guia pondera que, em alguns casos, o processamento mínimo pode influenciar negativamente no teor nutricional dos alimentos. Os exemplos são o arroz branco, em relação ao integral, e a farinha de trigo refinada, em relação à integral. Corroborando essas ponderações, o processo de transformação da fruta in natura em polpa (uma forma de processamento mínimo) há uma redução significativa do conteúdo de fibras para frutas como goiaba e uva ${ }^{[11]}$.

Assim, a contribuição da produção e do consumo de butiá (Butia yatai LORENZI \& NOBLICK) para a Segurança Alimentar e Nutricional é significativa na medida em que carrega uma série de elementos sociais, como os diferentes usos e significados locais, e ambientais, pela preservação e valorização da biodiversidade. $\mathrm{O}$ aspecto nutricional não deixa de ser relevante, pois como a comercialização de frutas nativas, inclusive a do butiá, ocorre por meio da produção de polpa congelada, é necessário averiguar se o processo de despolpa do butiá influi significativamente no teor de fibras. Por outro lado, também cabe refletir como a armazenagem influi na qualidade deste alimento, como por exemplo, se há variação do teor de ácido ascórbico longo da estocagem.

Portanto, esse trabalho teve como objetivo avaliar se o processo de despolpa de butiá interfere na composição, particularmente na densidade de energia do alimento, no teor de fibras e na estabilidade do ácido ascórbico durante o armazenamento da polpa.

\section{MATERIAL E MÉTODOS}

Foram utilizados frutos de butiá da safra de 2017, oriundos do município de Giruá (RS). As frutas foram colhidas no mês de março, limpas e congeladas. As análises 
ocorreram entre os meses de abril e junho deste mesmo ano.

Para o processamento as frutas foram descongeladas sob refrigeração por $24 \mathrm{~h}$ e divididas em dois lotes com $5 \mathrm{~kg}$ cada. A primeira parte foi submetida à retirada manual do caroço, simulando o processo de mastigação da fruta. O segundo lote foi processado em despolpadeira vertical, com 0,75 $\mathrm{HP}$ de potência e com malha de $1 \mathrm{~mm}$, para obtenção da polpa. Cerca de $200 \mathrm{~g}$ desta polpa obtida foi dividida em 4 porções e armazenada para realização das análises de estabilidade do ácido ascórbico.

Foram determinados parâmetros rendimento de extração, sólidos totais, sólidos solúveis ( ${ }^{\circ}$ Brix), $\mathrm{pH}$ e acidez total (\% ácido cítrico), lipídios, proteínas, fibras alimentares totais, carboidratos e cinzas com base na metodologia do Instituto Adolfo Lutz ${ }^{[12]}$.

As médias dos resultados foram comparadas entre si utilizando teste de Tukey a 5\% de significância. Todas as análises foram realizadas em triplicata, sendo apresentada a sua média juntamente com seu desvio padrão.

\section{RESULTADOS E DISCUSSÃO}

Os resultados de rendimento obtidos neste trabalho (Tabela 1) são similares aos obtido Tonietto et al. ${ }^{[13]}$ ao processar frutos de butiá oriundo de Tapes e Barra do Ribeiro, provavelmente da espécie Odorata ${ }^{[14]}$. Os autores identificaram rendimentos entre $72,3 \%$ e $89,6 \%$ para a polpa despolpada manualmente e valores entre $42,6 \%$ e $59,0 \%$ para fruta processada em despolpadeira. Entretanto, a peneira utilizada por Tonietto et al. ${ }^{[13]}$ propicia uma extração maior de polpa pois possui malha de $1,5 \mathrm{~mm}$, gerando polpas com rendimento e viscosidade distintas à polpa obtida neste trabalho ${ }^{[15]}$.
Tabela 1. Rendimento do butiá (Butia yatai) despolpado manualmente (Fruta) e na despolpadeira (Polpa)

\begin{tabular}{|c|c|c|}
\hline Parâmetro & Fruta & Polpa \\
\hline Rendimento (\%) & 71,9 & 36,5 \\
\hline
\end{tabular}

Os resultados físico-químicos (Tabela 2) indicam que a polpa apresenta maior teor de umidade, de açúcares dissolvidos e teor de ácido cítrico. Todavia, não houve diferença significativa na relação de açúcares e acidez (SS/AT), indicando semelhante aceitação sensorial.

Tabela 2. Parâmetros físico-químicos da fruta e da polpa de butiá (Butia yatai).

\begin{tabular}{|c|c|c|}
\hline Parâmetros & Fruta & Polpa \\
\hline Umidade (\%) & $80,7^{\mathrm{b}} \pm 0,1$ & $83,3^{\mathrm{a}} \pm 0,1$ \\
\hline $\begin{array}{c}\text { Sólidos Solúveis } \\
\left({ }^{\mathrm{o}} \text { Brix }\right)\end{array}$ & $11,5^{\mathrm{b}} \pm 0,4$ & $12,7^{\mathrm{a}} \pm 0,4$ \\
\hline $\mathrm{pH}$ & 3,4 & 3,4 \\
\hline $\begin{array}{c}\text { Acidez Total } \\
(\% \text { de ácido } \\
\text { cítrico) }\end{array}$ & $1,3^{\mathrm{b}} \pm 0,0$ & $1,5^{\mathrm{a}} \pm 0,0$ \\
\hline Relação SS/AT & $8,7^{\mathrm{a}} \pm 0,1$ & $8,4^{\mathrm{a}} \pm 0,3$ \\
\hline
\end{tabular}

Médias seguidas por letras distintas são diferentes entre si pelo Teste de Tukey ( $5 \%$ de significância).

Com base nos dados obtidos, foi possível identificar que houve diferença significativa na concentração dos componentes químicos dissolvidos na polpa e na fruta. Os resultados de sólidos solúveis (SS) e pH deste trabalho estão de acordo com os obtidos por Nunes et al. ${ }^{[16]}$ que obtiveram valores entre 12,0 e $18,0{ }^{\circ}$ Brix e de $\mathrm{pH}$ entre 3,1 e 3,8. Por outro lado, os teores ácido cítrico foram menores em relação a estes mesmos autores que encontraram valores entre 1,8 e 3,9 g de ácido cítrico em 100 gramas de polpa. Ambas as amostras de butiá analisadas apresentaram valores maiores de SS em relação ao Butiaeriospatha analisado por Dal Magro et al. ${ }^{[17]}$, pois estes identificaram 
valores de 6,4 e $7,7^{\circ}$ Brix e de 2,93 e 3,06 para o $\mathrm{pH}$.

Apesar de recomendar o consumo de minimamente processado, o Guia Alimentar para População Brasileira também pondera que este processo pode desbalancear nutricionalmente a composição do alimento. Por exemplo, o polimento do arroz incorre na redução de 65,6\% na quantidade de fibras, segundo os dados da Tabela de Análise Centesimal de Alimentos $(\mathrm{TACO})^{[18]}$. Salgado et al. ${ }^{[1]]}$ também identificaram variações significativas no teor de fibras para diversas frutas, entre elas cabe destacar a goiaba e uva com $61,0 \%$ e 50,66\% de redução cada.

Os resultados centesimais (Tabela 3) indicam que houve significativa diferença nutricional entre os dois produtos. Em relação à fruta, a polpa apresentou um aumento de 19,6\% na quantidade energética total, o que está diretamente relacionado ao aumento de $84,8 \%$ no teor de lipídios totais e na redução de 47,9\% de fibras alimentares totais. Este resultado indica que a despolpa concentrou os compostos químicos dissolvidos na emulsão, principalmente os lipídios. Por outro lado, a diminuição do teor de fibras na polpa ocorre, pois boa parte da fibra e fica retida na despolpadeira.

Tabela 3. Composição centesimal da fruta e da polpa de butiá (Butia yatai).

\begin{tabular}{|c|c|c|}
\hline $\begin{array}{c}\text { Ponteúdo Calórico } \\
\text { (Kcal/100g) }\end{array}$ & Fruta & Polpa \\
\hline $\begin{array}{c}\text { Carboidratos } \\
\text { (g/100g de polpa) }\end{array}$ & $8,0^{\mathrm{b}} \pm 0,3$ & $54,0^{\mathrm{a}} \pm 1,1$ \\
\hline $\begin{array}{c}\text { Proteínas } \\
\text { (g/100g de polpa) }\end{array}$ & $0,5^{\mathrm{a}} \pm 0,0$ & $0,6^{\mathrm{a}} \pm 0,1$ \\
\hline $\begin{array}{c}\text { Lipídios } \\
\text { (g/100g de polpa) }\end{array}$ & $1,0^{\mathrm{b}} \pm 0,1$ & $1,9^{\mathrm{a}} \pm 0,1$ \\
\hline $\begin{array}{c}\text { Cinzas } \\
\text { (g/100g de polpa) }\end{array}$ & $0,7^{\mathrm{b}} \pm 0,0$ & $0,8^{\mathrm{a}} \pm 0,0$ \\
\hline $\begin{array}{c}\text { Fibras Alimentares } \\
\text { (g/100g de polpa) }\end{array}$ & $9,0^{\mathrm{a}} \pm 0,1$ & $4,7^{\mathrm{b}} \pm 0,2$ \\
\hline
\end{tabular}

Médias seguidas por letras distintas são diferentes entre si pelo Teste de Tukey (5\% de significância).
Exceto o alto teor de fibras alimentares, os demais resultados centesimais obtidos neste trabalho foram similares quando comparados a outros autores. Faria et al. ${ }^{[2]}$ observaram que o Butiacapitata apresentou 85,4\% de umidade, 0,3\% de proteínas, $2,6 \%$ de lipídeos, $0,9 \%$ de cinzas, $10,1 \%$ de fibras totais e $10,8 \%$ de carboidratos. Berskow et al. ${ }^{[19]}$ pesquisando a polpa de diferentes genótipos de Butiaodorata, obtiveram valores um pouco menores em relação ao teor de fibra alimentar, variando entre 1,05 e 3g a cada 100 gramas de polpa. Pesquisando esta mesma espécie de butiá, Ferrão et al. ${ }^{[20]}$ observaram valores menores de fibra bruta (entre $0,84 \%$ e $4,02 \%$ ) e de cinzas (entre $0,47 \%$ e $0,77 \%$ ), superiores em relação ao teor de proteínas $(0,57 \%-0,93 \%)$ e similares de lipídeos (0,37\%-2,27\%) para a composição da polpa.

Os resultados nutricionais obtidos também foram analisados à luz da RDC 54/2012, que regula a Informação Nutricional Complementar ${ }^{[21]}$. Ainda que essa RDC verse somente sobre alimentos embalados, os parâmetros deste documento foram tomados como referência para a fruta também. Nesse sentido, ambos os alimentos podem ser considerados de baixo teor de gorduras (menor que $3 \mathrm{~g} / 100 \mathrm{~g}$ ). Apesar do processo de despolpa ter reduzido significativamente $\mathrm{o}$ teor de fibras alimentares, tanto a fruta quanto a polpa podem ser consideradas alimentos ricos em fibras alimentares. A polpa pode ser classificada como "Fonte" (mínimo de $3 \mathrm{~g} / 100 \mathrm{~g})$ e a fruta como "Alto conteúdo" (mínimo de $6 \mathrm{~g} / 100 \mathrm{~g}$ ) de fibras.

A Tabela 4 identifica os dados referentes aos teores de ácido ascórbico da polpa de butiá ao longo de três meses de armazenamento. Foi possível perceber que houve uma redução significativa, porém gradativamente menor ao longo do tempo. 
Tabela 4. Variação da vitamina C da polpa congelada de butiá (Butia yatai) durante o armazenamento

\begin{tabular}{|c|c|}
\hline Mês & Ácido ascórbico $\mathbf{~ m g / 1 0 0 g )}$ \\
\hline Março/17 & $72,8^{\mathrm{a}} \pm 1,2$ \\
\hline Após 1 mês & $53,9^{\mathrm{b}} \pm 0,9$ \\
\hline Após 2 meses & $43,8^{\mathrm{c}} \pm 1,1$ \\
\hline Após 3 meses & $40,3^{\mathrm{d}} \pm 0,7$ \\
\hline
\end{tabular}

Médias seguidas por letras distintas são diferentes entre si pelo Teste de Tukey (5\% de significância).

Foi observada a redução de $44,6 \%$ do valor inicial de ácido ascórbico após os três meses de estocagem. $\mathrm{O}$ decaimento deste nutriente ao longo de cada mês, em relação ao mês anterior, foi de $26,0 \%, 18,7 \%$ e $8,0 \%$. A partir dos dados obtidos, foi possível estimar que cerca de $1 / 3$ do teor inicial desta vitamina permanece no produto final após um ano de estocagem.

Os dados estão de acordo com Bertin et al. ${ }^{[22]}$ que ao revisar os dados bibliográficos sobre estabilidade de vitaminas, também identificaram o decaimento da vitamina $C$ durante a estocagem em diferentes pesquisas. Foram encontradas perdas de $43 \%$ na polpa in natura de acerola após quatro meses de congelamento e $92 \%$ em caju-do-cerrado após 90 dias de estocagem. As autoras indicam que o processamento térmico prévio auxilia na retenção da vitamina $\mathrm{C}$ ao longo do armazenamento, pois também tem a função de inativar atividade enzimática endógena.

Cabe destacar a que ANVISA estabelece que a Ingestão Diária Recomendada (IDR) de Vitamina C (ácido ascórbico) é de $45 \mathrm{mg}^{[23]}$. Para um alimento ser identificado como "Alto teor de" ácido ascórbico é necessária a presença de, no mínimo, 25\% da IDR $(11,25 \mathrm{mg} / 100 \mathrm{~g}$ de produto) deste nutriente ${ }^{[24]}$ (BRASIL, 2012). Portanto, a polpa de BY analisada pode ser identificada como "Alto teor de ácido ascórbico" porque apresenta valores superiores aos necessários para tal, ainda que tenha sido possível identificar uma redução significativa dessa vitamina ao longo da estocagem.

Os resultados de ácido ascórbico estão de acordo com os obtidos por outros autores. Ao pesquisar a quantidade de ácido ascórbico presente na polpa de Butiacapitata, Krolow et al. ${ }^{[25]}$, Pereira et al. ${ }^{[2]}$ e Jacnaet al. ${ }^{[27]}$ encontraram valores de 46,9mg/100g, 32,0mg/100g e $70,2 \mathrm{mg} / 100 \mathrm{~g}$.

\section{CONCLUSÃO}

Portanto, com base nos dados obtidos nesse trabalho, foi possível identificar um aumento significativo na densidade energética da polpa de butiá yatai em relação. Também foi identificado que o processo de despolpa influencia negativamente na quantidade de fibras alimentares quando comparada a fruta e a polpa de butiá yatai.

Entretanto, ainda que haja um aumento do teor de lipídios e uma redução do teor de fibras alimentares, essa variação é menos significativa em relação ao polimento do arroz ou mesmo o refino da farinha de trigo. Assim, polpa de butiá representa uma importante opção de alimento saudável, pois além de ser um produto da biodiversidade local, carregado de sentidos culturais e históricos, ainda pode ser considerado fonte de fibras e de vitamina C. Além disso, o uso da polpa de butiá também se justifica pela maior facilidade de consumo, permitindo maior consumo per capita.

Portanto, tal como indica o Guia Alimentar para População Brasileira, ambos os alimentos devem compor a base $\mathrm{da}$ alimentação regional. Tanto a fruta quanto a polpa podem ser entendidos como alimentos saudáveis de forma ampla, pois ao mesmo tempo em que apresentam boas características nutricionais, o consumo destes alimentos também pode fortalecer laços socioculturais, valorizando a biodiversidade e preservando os recursos ambientais. 


\section{AGRADECIMENTOS}

À Cadeia Solidária das Frutas Nativas pelo apoio. A CAPES e Pró-Reitoria de Extensão da UFRGS pelo financiamento.

\section{REFERÊNCIAS}

[1] Hoffman JF, Barbieri RL, Rombaldi CV, Chaves, FC. Butiaspp. (Aracaceae): An overview. ScientiaHorticulturae. n. 179: 122-131, 2014.

[2] Faria JP, Almeida F, Silva LCR, Vieira RF, AgostiniCosta TS. Caracterização da polpa do coquinho-azedo (Butacapitata var capitata). Rev. Bras. Frutic., Jaboticabal - SP, v. 30, n.3, p.827-829, Setembro, 2008. [internet]. [Acesso em 15 fev. 2017]. Disponível em: http://www.scielo.br/scielo.php?script=sci arttext\&pi $\underline{\mathrm{d}=\mathrm{S} 0100-29452008000300045}$

[3] Food and Agriculture Organizations. Sustainable Diets and Biodiversity.Roma, 2010. [internet]. [Acesso em 15 fev 2018]. Disponível em http://www.fao.org/docrep/016/i3004e/i3004e.pdf

[4] Takeuti D, Oliveira JM. Para além dos aspectos nutricionais: uma visão ambiental do sistema alimentar. Segurança Alimentar e Nutricional, Campinas, 20(2):194-203, 2013. Disponível em https://periodicos.sbu.unicamp.br/ojs/index.php/san Larticle/view/8634597/2518

[5] Barbieri RL. Vida no Butiazal. Brasília (DF), Embrapa, 2015. Disponível em: https://www.embrapa.br/noticias-rss/Lasset publisher/HA73uEmvroGS/content/id/21473 $\underline{788}$

[6] Buttow MV, Barbieri RL, Neitzke RS, Heiden G. Conhecimento Tradicional Associado ao uso de Butiás (Butiaspp.,Arecaceae) no sul do Brasil. Rev. Bras. Frut., Jaboticabal - SP, v. 31, n. 4, p.1069-1075, Dezembro 2009. Disponível em: http://www.scielo.br/scielo.php?pid $=\mathrm{S} 0100-$ $29452009000400021 \&$ script $=$ sci abstract\&tlng $=$ pt

[7] Kohler M, Brack P. Frutas nativas no Rio Grande do Sul: Cultivando e valorizando a diversidade. Revista Agriculturas, v. 13, n. 2, junho 2016. Disponível em: http://aspta.org.br/wpcontent/uploads/2016/08/Agriculturas V13N2Artigo01.pdf
[8] Brasil. Ministério da Saúde. Secretaria de Atenção à Saúde. Departamento de Atenção Básica. Guia Alimentar para População Brasileira, $2^{\mathrm{a}}$ edição. Brasília (DF), 2014. Disponível em: http://bvsms.saude.gov.br/bvs/publicacoes/gui a_alimentar_populacao_brasileira_2ed.pdf

[9] Monteiro CA, Levy RB, Claro RM, Castro IRR, Cannon G. A new classification of foods based on the extend and purpouse of their processing. Cad. Saúde Pública, Rio de Janeiro, 26(11):2039- 2049, nov, 2010. Disponível em: http://www.scielo.br/scielo.php?script=sci artt ext\&pid=S0102-311X2010001100005

[10] Monteiro CA, Cannon G, Levy RB, Moubarac JC, Laime P, Martins AP, Canella D, Louzada M, Parra D. NOVA The star shinesbright. Wolrd Nutrition Volume 7, number 1-3, january-march 2016. Disponível em: $\quad$ http://archive.wphna.org/wpcontent/uploads/2016/01/WN-2016-7-1-3-28-

38-Monteiro-Cannon-Levy-et-al-NOVA.pdf

[11] Salgado SM, Guerra NB, Melo Filho AB. Polpa de fruta congelada: Efeito do processamento sobre o conteúdo de fibra alimentar. Rev. Nutr., Campinas, 12(2): 303-308, set/dez, $1999 . \quad$ Disponível em: http://www.scielo.br/pdf/rn/v12n3/v12n3a09

[12] Instituto Adolfo Lutz. Métodos físicoquímicos para análise de alimentos. Versão eletrônica, São Paulo, 2008. Disponível em: http://www.ial.sp.gov.br/resources/editorinplac e/ial/2016 3 19/analisedealimentosial 2008.pd $\underline{\mathrm{f}}$

[13] Tonietto A, Schlindwein G, Tonietto SM, Bezerra AES, Duprat ACD, Costa AA. Qualidade de polpa de butiá obtida por processamento industrial. In. XX Congresso Brasileiro de Fruticultura. 54th Annual Meeting of the Interamerican Society for Tropical Horticulture.Centro de convenções, Vitória (ES), 2008.

[14] Eslabão MP, Pereira PEE, Barbieri RL, Heiden G. Mapeamento da distribuição geográfica de butia (arecaceae). In: $18^{\circ}$ Encontro 
de pós-graduação UFPEL. Pelotas, 2016. Disponível em: https://www.embrapa.br/busca-de-publicacoes/Lpublicacao/1058641/mapeamento-da-distribuicaogeografica-de-butia-arecaceae

[15] Martins JS, Melo EM, Cardoso FD, Ramos MO, Hertz, PF. Comparação de rendimento e de parâmetros físico-químicos entre diferentes formas de processamento de polpa de Butiacatarinensis. Cadernos de Agroecologia, v. 13, n. 1, 2018.

[16] Nunes AM, Fachinello JC, Radmann EB, Bianchi VJ, Schwartz E. Caracteres Morfológicos e Físicoquímicos de butiazeiros (Butiacapitata) na região de Pelotas, Brasil. Interciência, Vol. 35, n. 7, p. 500-505, Jul 2010. Disponível em: http://www.redalyc.org/pdf/339/33914381005.pdf

[17] Dal Magro NG, Coelho SRM, Haida KS, Berté SD, Moraes SS. Comparação físico-química de frutos congelados de Butiaeriospatha (Mart.) Becc. Do Paraná e Santa Catarina (BR). Revista Varia Scientia v.06, n.11, p.33-42, 2006. Disponível em: http://erevista.unioeste.br/index.php/variascientia/article/vie $\underline{\mathrm{w} / 704 / 594}$

[18] Brasil. Tabela Brasileira de Composição de alimentos/NEPA-UNICAMP. Campinas, 2004. Disponível em: http://www.cfn.org.br/wpcontent/uploads/2017/03/taco 4 edicao ampliada e revisada.pdf

[19] Berskow GT, Hoffmann JF, Teixeira AM, Fachinello JC, Chaves FC, Rombaldi CV. Bioactive and yield potential of jelly palms (Butia odorata Barb. Rodr.) Food Chemistry 172: 699-704, 2015. Disponível em:

https://www.sciencedirect.com/science/article/pii/S0 308814614014964?via\%3Dihub

[20] Ferrão TS, Ferreira DF, Flores DW, Bernardi G, Link D, Barin J, Wagner R. Evaluation of composition and quality parameters of jelly palm (Butia odorata) fruits from different regions of Souther Brazil. FoodResearchInternational 54: 57-62, 2013.

[21] Brasil. Agencia Nacional de Vigilância Sanitária. Regulamento Térmico sobre Informação Nutricional Complementar. Resolução de Diretoria Colegiada (RDC) n 54, de 12 de novembro de 2012. Disponível em:

http://portal.anvisa.gov.br/documents/\%2033880/25 68070/rdc0054 1211 2012.pdf/c5ac23fd974e-4f2c-9fbc-48f7e0a31864

[22] Bertin RL, Schulz M, Amante ER. Estabilidade de vitaminas no processamento de alimentos: uma revisao. B.CEPPA, v. 34, n. 2, jul/dez, 2016. Disponível em: http://revistas.ufpr.br/alimentos/article/view/5 $\underline{3177 / 32560}$

[23] Brasil. Agencia Nacional de Vigilância Sanitária. Regulamento Técnico sobre a ingestão diária recomendada (IDR) de proteína, vitamina e minerais. Resolução de Diretoria Colegiada (RDC) $n^{\circ} 269$ de setembro de 2005. Disponível em:

http://portal.anvisa.gov.br/documents/33916/3 94219/RDC_269 2005.pdf/2e95553c-a48245c3-bdd1-f96162d607b3

[24] Krolow ACR, Vizzotto M, Barbieri RL, Fonseca

LX.

ProcessingandcharacterizationofButiacapitatafro

m Rio Grande do Sul. InternationalConferenceonFoodInnovation, Universidad Politécnica de Valencia, 2010.

[25] Pereira MC, Steffens RS, Jablonski A, Hertz $\mathrm{PF}$, Rios AO, Vizzotto M, Flores SH. Characterizations, bioactive compounds and antioxidant potential of three Brazillian fruits. Journal of Food Composition and Analysis n. 29: 19-24, 2013.

[26] Jacna TJ, Hermes VS, Flores SH, Rios AO. Bioactive compounds in pindo palm (Butiacapitata) juice and in pomace resulting of the extration process. J SciFoodAgric n. 96: 1216-1222, 2016. 